\title{
Quantization conditions for the periodic Toda chain: Inadequacy of Bethe-ansatz methods
}

\author{
Michael Fowler and Holger Frahm \\ Department of Physics, University of Virginia, Charlottesville, Virginia 22901
}

(Received 19 December 1988)

\begin{abstract}
Gutzwiller has developed a scheme for determining the energy levels of a finite quantum Toda lattice. We present a numerical analysis using his method and calculate low-lying energy levels for some small lattices. We check the completeness of his quantization conditions in the harmonic (low-energy) and the semiclassical (high-energy) limits. Our main finding is that the Bethe-ansatz spectrum equations, known to be exact for an infinite Toda lattice in the classical limit, are incorrect for finite and quantum lattices.
\end{abstract}

\section{INTRODUCTION}

The Toda lattice is a nonrelativistic one-dimensional gas of point particles of equal mass $m$ interacting via an exponential nearest-neighbor potential of strength $V$ and range $1 / \gamma$,

$$
H=\frac{1}{2 m} \sum_{i} p_{i}^{2}+V \sum_{i} e^{\gamma\left(q_{i+1}-q_{i}\right)} .
$$

Note that the force only operates between nearest neighbors, in contrast to forces between particles in standard one-dimensional-gas models. This is a lattice system visualized in terms of balls with springs between them, although the springs are nonphysical in that they exert a rapidly weakening force as the particles separate. Toda ${ }^{1,2}$ constructed this Hamiltonian by requiring complete integrability of a nontrivial (classical) lattice system. It turns out that the quantum system is also integrable, ${ }^{3}$ having phonon and soliton modes, and is close to a sineGordon system with low but nonzero winding-number density. ${ }^{4}$

In the present paper, we address the quantum properties of this system. Our study was motivated by the puzzling observation that, at least for a large Toda lattice in the classical limit, the Bethe-ansatz method applied by Sutherland ${ }^{5}$ and Opper ${ }^{6}$ describes the ground-state energy, low-energy-excitation spectrum, and thermodynamics apparently precisely, even though the wave function manifestly cannot be of the Bethe-ansatz form, since the potential has a finite decay length. We wanted to find out if the Bethe-ansatz spectrum equations are exact for all Toda lattices. Our work is based largely on the work of Gutzwiller, ${ }^{7}$ who constructed (thereby extending his earlier work on the two- and three-body closed Toda chain ${ }^{8}$ ) explicit series representations of wave functions for up to four particles that allow for straightforward generalization to larger systems. However, he only provided plausibility arguments for a set of quantization criteria, and did not actually find the energy levels. We have succeeded in numerically analyzing these small systems using Gutzwiller's techniques. We find his quantization criteria do indeed give the correct spectrum in a regime that can be checked by other means - the low-energy quasiclassical limit, where the system becomes a harmonic chain. Furthermore, over a wider energy range we find the Gutzwiller energy levels to be very close to those given by semiclassical quantization of the canonical dynamical variables. This strongly suggests that Gutzwiller's criteria are correct. On the other hand, the Bethe-ansatz does not give the energy levels nearly so accurately, and in fact misses a large fraction of the total number of states. This is to say, for a finite Toda lattice, the Betheansatz predictions of energy levels are definitely incorrect. This should be contrasted with, for example, a finite system of particles interacting via an inverse-square potential, where again the wave function is not of Betheansatz form, but the Bethe-ansatz equations do give the energy levels exactly. We should add, though, that in the limit of a large system, the Bethe-ansatz results become accurate. For example, in the classical limit the error in the Bethe-ansatz prediction of the ground-state energy is of order $N^{-3}$ per particle for a system of $N$ particles. Also, the omission of a fraction of the states does not affect the classical thermodynamics, being equivalent to a renormalization of $\hbar$, and therefore disappearing in the limit of $\hbar \rightarrow 0$. Thus our results are fully consistent with the previous work of Sutherland and Opper. ${ }^{5,6}$

We now briefly survey the history of the Toda lattice to place our work in context. The classical system was first introduced by Toda and has the unusual property of being completely integrable both for the open (infinite line) and closed (on a ring) systems. In fact, a simple argument due to Flaschka ${ }^{9}$ gives $N$ separately conserved quantities for the $N$-particle open system. Flaschka's argument is reviewed in the next section. The main point is that if for the open system the initial state has the particles widely separated, with momenta $\lambda_{1}, \ldots, \lambda_{N}$ then the final state after all collisions has the same set of momenta, and in fact this set $\left\{\lambda_{i}\right\}$ are the eigenvalues of an isospectral matrix whose elements (given explicitly below) are functions of the momenta and positions of $N$ particles. Thus throughout the motion there are $N$ separately conserved quantities given by $\sum_{i} \lambda_{i}^{n}, n=1, \ldots, N$. In contrast, the periodic or closed system has, of course, no sim- 
ple final state, and proved more difficult to analyze. A complete solution of the classical problem for the closed system was presented by $\mathrm{Kac}$ and van Moerbeke, ${ }^{10}$ and was actually based on an extension of Flaschka's work. They considered the open $(N-1)$-particle subsystem given by removing one particle from the system, and relabelled the $\left\{\lambda_{i}\right\}$ for this subset as $\mu_{1}, \ldots, \mu_{N-1}$. Under the full $N$-particle Hamiltonian the $\left\{\mu_{i}\right\}$ are no longer invariant, but exhibit relatively simple behavior - they are a set of commuting variables (in the Poisson bracket sense) and move in nonoverlapping closed paths. The canonically conjugate variables are labelled $v_{i}$. It is also necessary to include the total system momentum and center-of-mass position to get a complete set of dynamical variables. Kac and van Moerbeke were able to integrate the equations of motion for the $\mu_{i}$ and thus solve the classical Toda problem.

A first attempt to consider quantum effects in the Toda lattice was made by Shirafuji. ${ }^{11}$ Starting from the wellknown one-soliton solution of the classical periodic Toda chain, he obtained semiclassical corrections to the classical energy in the Wentzel-Kramers-Brillouin (WKB) approximation. However, it appears difficult to apply his approach to excitations other than the single soliton, or to get higher-order terms in $\hbar$.

The first work on the quantum Toda lattice was the Bethe-ansatz analysis by Sutherland. ${ }^{5}$ For a dilute open lattice, the asymptotic wave function is correctly described by the Bethe ansatz, because the many-particle scattering matrix is factorizable so no diffraction occurs. Of course, the true-system wave function will deviate from the plane-wave Bethe-ansatz form when the particles are close enough for the interaction to be significant, but this is a small fraction of phase space for the dilute system. It should be noted that the momentum parameters appearing in the Bethe wave function will be just the set $\left\{\lambda_{i}\right\}$ from Flaschka's analysis. The surprising result in Sutherland's work was successful derivation of the known classical results for the ground-state energy and excitation spectrum of a large closed lattice. The Bethe ansatz yields the correct result for this case even for densities of order unity, that is, one particle per decay length of the potential. ${ }^{12}$ In this case, the system spends most of its time in a region of phase space where the Bethe-ansatz wave function is manifestly wrong. Later, Opper ${ }^{6}$ applied the standard Bethe-ansatz thermodynamic analysis to demonstrate, equally surprisingly, that Sutherland's approach also gave the free energy of the closed classical system correctly in the appropriate $\hbar \rightarrow 0$ limit.

These results made it appear possible that, despite the incorrectness of the wave function, the Bethe-ansatz equations for the energy levels might be exactly correct for the closed Toda lattice. Indeed, Sutherland had previously found this to be the case for an apparently very similar system, that of particles on a line interacting via an inverse-square potential. ${ }^{13}$ That system is integrable both classically and quantum mechanically, the wave functions do not have Bethe-ansatz form, yet for the closed system the Bethe ansatz equations give the spectrum exactly for any total number of particles. In light of this result, it seemed conceivable that the requirement of full integrability so severely constrained the dynamics of a quantum system that even if the wave functions were not quite of Bethe-ansatz form, the Bethe-ansatz energy levels might still be exactly correct. However, there is an important difference between Sutherland's $1 / r^{2}$ system and the periodic Toda chain ${ }^{3}$ : The integrability of both of them can be understood as a consequence of a hidden underlying symmetry, generated by the finite-dimensional Lie algebra $\mathrm{SU}(N)$ in the $N$-particle inverse-square system (and the open Toda chain) and an infinitedimensional Kac-Moody algebra in the closed Toda chain. While the Hamiltonian can be represented as the projection of that of free motion in a higher-dimensional curved space in the case of a finite-dimensional symmetry (where the degrees of freedom are trivially separated), this is not possible in the second. This may be the reason for the fact mentioned above and discussed in detail below: In contrast to the inverse-square systems, we find that for a finite, closed Toda lattice the energy levels given by the Bethe ansatz are definitely incorrect, although they do approach the right ones asymptotically as the size of the system increases.

The first, systematic, quantum-mechanical treatment of the closed Toda system from first principles was the work of Gutzwiller, ${ }^{7,8}$ on which the present paper is based. Gutzwiller's basic strategy was to try to mimic the successful classical analysis of $\mathrm{Kac}$ and van Moerbeke. That is to say, he cut open the $N$-body closed Toda chain by removing one particle, and solved the open $(N-1)$-body problem. Then, he incorporated the variables describing the asymptotic momenta of the open $(N-1)$-body system into the dynamical description of the closed $N$-body chain. In the classical analysis, these were the $\left\{\mu_{i}\right\}$, which underwent periodic motion in the closed system. For the quantum case, the wave function for the closed system can be written as a sum over wave functions for the open $(N-1)$-body system (with an extra plane-wave factor for the $N$ th particle to give total momentum zero) where the allowed sets of momenta are of the form $\left\{\rho_{j}+i n_{j} \hbar\right\}$ with the $\rho_{j}$ real (analogous to $\mu_{j}$ above) and the $n_{j}$ integers ranging from $+\infty$ to $-\infty$. Thus, the wave function is defined by the set of momenta $\left\{\rho_{j}\right\}$ and the $(N-1)$-dimensional integer grid of coefficients of the plane-wave terms. The Schrödinger operator is a difference operator in one direction on this grid (note that the potential-energy term $e^{q_{2}-q_{1}}$ increases $n_{2}$ by unity, and decreases $n_{1}$ by unity when acting on the wave function). The other conserved quantities give difference operators in other directions, and using them all one can construct the table of coefficients. If we set as boundary conditions on this process that the series must converge on summing over negative values of the integers $n_{j}$, then in general the coefficients generated will not be such that the contribution to the wave function from summing over terms with positive $n_{j}$ converges. That is to say, for the wave function to be well defined at each point in configuration space, $N-1$ parameters must be fixed in order to link up the convergent $n_{j} \rightarrow-\infty$ sequences with the convergent $n_{j} \rightarrow+\infty$ sequences. It turns out that this fixes the set of real momenta $\left\{\rho_{j}\right\}$ for given values of 
the energy and the other conserved quantities. Actual numerical computation of $\left\{\rho_{j}\right\}$ may, however, be difficult, especially near the classical limit. Once the appropriate $\rho_{j}$ are inserted, the wave function is well defined everywhere in configuration space. This does not mean it is normalizable - in general, it will have exponentially increasing terms into the repulsive potentials. To ensure normalizability, these terms must have zero coefficients, and this requirement leads to the quantization condition for the actual eigenstates of the system. These are Gutzwiller's quantization conditions mentioned earlier, and discussed in detail in Sec. III. Despite their simple appearance, they are not trivial to derive, and indeed the second one is rather conjectural. An important practical point in using these conditions is that Gutzwiller's function $\phi(\rho)$ (see below) is rather flat for $\rho$ close to $\rho_{j}$, so precise evaluation of $\rho_{j}$ is not essential to finding the spectrum with good accuracy. At the end of Sec. III, we discuss some technical points connected with the symmetry of the ground state for the odd-particlenumber system.

It is interesting to note that the quantum analysis outlined above would not work for the inverse square system mentioned earlier, because in that case all particles interact, and removing one of them would not turn a closed system into an open one. In fact, the closed $r^{-2}$ system actually has a $[\sin (2 \pi r) / L]^{-2}$ potential, corresponding to a sum of $r^{-2}$ interactions at $r, r+L, r+2 L, \ldots$. These repetitions do not, of course, occur in the Toda system where the interaction is only between nearest neighbors.

In Sec. IV of this paper, we find the energy levels in the semiclassical regime by semiclassical quantization of the motion described by Kac and van Moerbeke ${ }^{10}$ in terms of the canonical variables $\mu_{i}$ and $v_{i}$. Our purpose is to verify that the energy levels found numerically using Gutzwiller's method are correct in this regime. We find the $\mu_{i}, v_{i}$ semiclassical quantization to be in excellent agreement with the Gutzwiller results, and, as expected, to have a much greater range of validity than the (lowenergy) harmonic chain approximation.

In Sec. $\mathrm{V}$ we review the Bethe-ansatz approach to the Toda lattice in some detail, and present results on the errors incurred in using Bethe-ansatz methods for finite lattices.

Finally, we should mention that the quantum-inversescattering method has been applied to the Toda lattice. The operator commutation relations were written down and discussed briefly by Gaudin, ${ }^{14}$ and a more detailed development was presented by Sklyanin. ${ }^{15} \mathrm{He}$ gave an elegant derivation of the difference form of Schrödinger's equation. However, we are not able to compare our results with his because he only analyzed the infinite chain, where he rederived the Bethe-ansatz results. For the finite chain, we have not succeeded in linking up his wave-function ansatz worth our work.

\section{THE CLASSICAL TODA LATTICE}

After rescaling of all positions by $1 / \gamma$ and momenta by $\sqrt{m V}$ we can write the Hamiltonian in the following dimensionless form (energies are measured in units of $V$ ):

$$
H=\frac{1}{2} \sum_{i} p_{i}^{2}+\sum_{i} e^{\left(q_{i+1}-q_{i}\right)} .
$$

As observed by Flaschka ${ }^{9}$ the dynamical equations for the periodic Toda Hamiltonian Eq. (1') can be written in the form

$$
\frac{d \mathcal{L}}{d t}=\frac{1}{2}(\mathcal{L} B-\mathscr{B} \mathcal{L})
$$

with $\mathcal{L}$ and $\mathcal{B}$ being the following pair of Lax matrices (for $N>2$ ):

$$
\begin{aligned}
& \mathcal{L}=\left(\begin{array}{ccccc}
b_{1} & a_{1} & 0 & \cdots & a_{N} \\
a_{1} & b_{1} & a_{2} & & \\
0 & a_{2} & b_{3} & & \\
\vdots & & & & \\
a_{N} & 0 & 0 & \cdots & b_{N}
\end{array}\right), \\
& \mathcal{B}=\left(\begin{array}{ccccc}
0 & a_{1} & 0 & \cdots & -a_{N} \\
-a_{1} & 0 & a_{2} & & \\
0 & -a_{2} & 0 & & \\
\vdots & & & & 0 \\
a_{N} & 0 & 0 & \cdots & 0
\end{array}\right) .
\end{aligned}
$$

Here $a_{n}=\exp \left[\left(q_{n+1}-q_{n}\right) / 2\right]$ and $b_{n}=p_{n}$. From the equation of motion (2) it is clear that the eigenvalues of $\mathcal{L}$ or, alternatively, the roots $\lambda_{1}<\lambda_{2}<\cdots<\lambda_{N}$ of the polynomial

$$
D(\lambda) \equiv \operatorname{det}|\lambda-\mathcal{L}|+2 \equiv \prod_{j=1}^{N}\left(\lambda-\lambda_{j}\right)
$$

do not change in time, i.e., they are integrals of motion that can be written as algebraic functions in $\left\{a_{n}, b_{n}\right\}$. Together with the $\lambda_{j}$ any combination of these quantities is conserved: In the following we will use the coefficients of $D(\lambda)$ is addition to $\left(\lambda_{j}\right)$ :

$$
\begin{aligned}
D(\lambda)= & \lambda^{N}-P \lambda^{N-1}+\left(P^{2} / 2-E\right) \lambda^{N-1} \\
& +A \lambda^{N-3}+B \lambda^{N-4}+\cdots
\end{aligned}
$$

where

$$
P=\sum_{j} \lambda_{j}=\operatorname{tr}(\mathcal{L})
$$

and

$$
E=\frac{1}{2} \sum_{j} \lambda_{j}^{2}=\frac{1}{2} \operatorname{tr}\left(\mathcal{L}^{2}\right)
$$

are the total momentum and energy, respectively. This representation of the periodic Toda chain when applied to the classical ground-state configuration ( $q_{n} \equiv$ const, $p_{n} \equiv 0$ ) gives

$$
\lambda_{j}=-2 \cos [\pi(2 j-1) / 2 N] \quad(j=1, \ldots, N)
$$

or $P=0, E=N, A=0, \cdots$.

The dynamics of the open Toda chain can be treated in a very similar way by eliminating the elements $a_{N}$, from the matrices $\mathcal{L}$ and $\mathscr{B}$ [this is equivalent to removing the 
term $\exp \left(q_{1}-q_{N}\right)$ from the Hamiltonian Eq. (1')]. However, in this case there exists a very simple physical interpretation of the eigenvalues of $\mathcal{L}$ : In the region of configuration space where the exponential potential is weak, namely $\exp \left(q_{i+1}-q_{i}\right) \ll 1$ for $i=1, \ldots, N-1$, the $a_{n}$ vanish and the eigenvalues of $\mathcal{L}$ are just the asymptotic momenta $b_{n}=p_{n}$ of the particles.

This simple picture does not apply to the periodic chain, but Kac and van Moerbeke ${ }^{10}$ used the $(N-1)$ particle open chain obtained by eliminating the first particle from the $N$-particle periodic one to construct a new set of canonically conjugate variables. After removing of the first row and the first column of the matrix $\mathcal{L}$ they considered the eigenvalues $\mu_{1}, \mu_{2}, \ldots, \mu_{N-1}$ of the resulting matrix $\mathcal{L}^{*}$. They were able to show that these eigenvalues are restricted each to one of the $N-1$ closed intervals where the polynomial $D(\lambda)$ satisfies $|D(\lambda)| \geq 2$.

The Hamiltonian in these $\mu_{n}$ and $N-1$ conjugate variables $v_{n}$ (obeying canonical Poisson brackets with the $\mu_{n}$ ) giving dynamical equations equivalent to (2) is given by

$$
H\left(\left\{v_{n}, \mu_{n}\right\}\right)=\sum_{i=1}^{N-1}\left(2 \cosh v_{i}-\mu_{i}^{N}\right) / m^{\prime}\left(\mu_{i}\right),
$$

where

$$
m^{\prime}(\mu)=\frac{d}{d \mu}\left[\prod_{i}\left(\mu-\mu_{i}\right)\right] .
$$

Furthermore, it can be shown that at any point in the phase space of the $\left\{v_{n}, \mu_{n}\right\}$ the relation

$$
2 \cosh v_{i}=\left|D\left(\mu_{i}\right)\right|
$$

holds with $D$ being the characteristic polynomial (4) of the matrix $\mathcal{L}$.

Together with the total momentum $\Sigma_{i} p_{i}$ and the center-of-mass position the new variables $\left\{v_{n}, \mu_{n}\right\}$ form an alternative set of canonical variables for the description of the periodic Toda lattice.

\section{QUANTIZATION OF THE PERIODIC TODA LATTICE}

Quantization of the Toda lattice is achieved by finding simultaneous (normalizable) eigenfunctions to the operator equivalents of the classical conserved quantities $P, E, A, B, \ldots$ [see Eq. (4b)]. Following the classical solution of the $N$-particle periodic Toda lattice by $\mathrm{Kac}$ and van Moerbeke, Gutzwiller ${ }^{7}$ first solved the $(N-1)$ particle open chain which gives a set of states defined by their asymptotic behavior in the part of configuration space where the particles are widely separated, i.e., $\exp \left(q_{i+1}-q_{i}\right)<<1$ for $i=2, \ldots, N-1$,

$$
\psi\left(q_{2}, \ldots, q_{N} ;\left\{k_{j}\right\}\right) \simeq \sum_{\pi}(-)^{\pi} \exp \left[i \sum_{j} k_{\pi(j)} q_{j}\right]
$$

$k_{j}$ being the wave number equivalents of the momenta $\mu_{j}$ in the classical limit. Using these as a basis he expanded the wave function for zero total momentum states in the periodic $N$-body Toda lattice in a series:

$$
\begin{aligned}
\Psi\left(q_{1}, q_{2}, \ldots, q_{N}\right)= & \sum_{\left\{\kappa_{j}\right\}} C\left(\left\{\kappa_{j}\right\}\right) e^{-i\left(\sum_{j} \kappa_{j}\right) q_{1}} \\
& \times \psi\left(q_{2}, \ldots, q_{N} ;\left\{\kappa_{j}\right\}\right) .
\end{aligned}
$$

The operators $P, E, A, B, \ldots$ are polynomials of $p_{j}$ and $e^{ \pm q_{j}}$, where the action of the latter on this wave function is a translation $\kappa_{j} \rightarrow \kappa_{j} \pm i$ [note that in our Hamiltonian (1') all the $p_{j}$ and $q_{j}$ and consequently $\hbar$ or, in terms of the physical parameters, $\gamma \hbar / \sqrt{m V}$ are dimensionless]. Hence, the summation in (8) has to be performed over the $N-1$ integers $n_{j}$ in $\kappa_{j}=k_{j}+i n_{j}$. With this ansatz. the Schrödinger equation separates into a set of second-order difference equations

$$
r_{n}(\kappa+i)+D(\hbar \kappa) r_{n}(\kappa)+r_{n}(\kappa-i)=0, \quad n=2, \ldots, N,
$$

where

$$
C\left(\left\{\kappa_{j}\right\}\right) \propto\left[\prod_{n>n^{\prime}}\left(\kappa_{n}-\kappa_{n^{\prime}}\right)\right] \prod_{n} r_{n}\left(\kappa_{n}\right)
$$

and $D(x)$ as given by Eq. (4). These equations have to be considered as an eigenvalue equation for $\kappa$. Having found a set of $\left\{\kappa_{j}\right\}$ the quantization of the periodic Toda chain is obtained by requiring normalizability of the wave function (8). The set of difference equations (9) has been obtained by Sklyanin ${ }^{15}$ in a similar approach using the framework of the quantum-inverse-scattering formalism.

It is worth noting that the ansatz (8) for the wave function is a generalization of the well-known result of Floquet theory for ordinary differential equations: for $N=2$ the open $(N-1)$-particle subsystem describes a free particle with eigenfunctions being plane waves $\psi\left(q_{2} ; k\right)$ $\propto \exp \left(i k q_{2}\right)$. The Schrödinger equation for the two-body periodic Toda chain, after separation of the center-ofmass motion, is the modified Mathieu equation ${ }^{16}$

$$
\left[\frac{d^{2}}{d x^{2}}-(a-2 b \cosh 2 x)\right] \psi(x)=0
$$

with $2 x=q_{2}-q_{1}, a=-4 E / \hbar^{2}$, and $b=-4 / \hbar^{2}$. As can be easily checked, these values of the parameters $a$ and $b$ lie in the first unstable region of the Mathieu equation. Hence, the Floquet ansatz for the solution of (10) coincides with Gutzwiller's ansatz (8) in the $N=2$ system:

$$
\Psi\left(q \equiv q_{2}-q_{1}\right)=e^{i k q} \sum_{n} c_{n} e^{-n q}
$$

Just as in the solution of the Mathieu equation, the values of the Floquet wave numbers $k_{j}$ are equal to the zeros of the Hill-type determinant (apart from a factor of $\hbar)$ 


$$
\Delta(\rho)=\left|\begin{array}{ccccc}
\ddots & & & & \\
\frac{1}{D(\rho-i \hbar)} & 1 & \frac{1}{D(\rho-i \hbar)} & & \\
& \frac{1}{D(\rho)} & 1 & \frac{1}{D(\rho)} & \\
& & \frac{1}{D(\rho+i \hbar)} & 1 & \frac{1}{D(\rho+i \hbar)}
\end{array}\right|,
$$

namely, $\Delta(\rho)=0$ for $\rho=\hbar k_{j}[D(\rho)$ is the characteristic polynomial of the classical Lax matrix as given by Eqs. (4), so for the two-particle case $D(\rho)=\rho^{2}-E$ ]. This condition guarantees that $\sum_{n=-\infty}^{\infty} r\left(k_{j}+i n\right)$ converges absolutely for one of the solutions of Eq. (9).

It is obvious that $\Delta$ is a periodic function with period $i \hbar$ going to 1 for $\rho \rightarrow \pm \infty$ and having simple poles at the zeros $\lambda_{j}(\bmod i \hbar)$ of $D(\rho)$ [Eq. $(4 a)$ ]. Generalizing Hill's argument $^{17}$ for infinite determinants of this type, Gutzwiller has shown that

$$
\Delta(\rho)=1+\pi \sum_{j=1}^{N} K_{j} \operatorname{coth}\left(\frac{\pi}{\hbar}\left(\rho-\lambda_{j}\right)\right)
$$

with $K_{j}$ being real functions of $\hbar$ and the set of conserved quantities $\left\{\lambda_{j}\right\}$.

In the semiclassical regime $\hbar<<1$ the dependence of $\Delta$ on $\rho$ along the real axis becomes particularly simple: Between the poles $\Delta$ takes constant values $\Delta_{j}\left(\Delta=\Delta_{j}\right.$ for $\left.\lambda_{j}<\rho<\lambda_{j+1}\right)$ while $\Delta \equiv 1$ for $\rho$ outside the interval $\left[\lambda_{1}, \lambda_{N}\right]$, since $\Delta$ equals one at infinity. This suggests that the zero $\rho_{j}$ of $\Delta$ is located in a neighborhood of $\lambda_{j}$ with radius of order $\hbar$. However, numerical calculations of $\Delta$ show that this is not the case since $\Delta_{j} \propto \exp \left(-2 \pi \delta_{j} / \hbar\right)$ as $\hbar \rightarrow 0$. This functional dependence on $\hbar$ results in a finite difference $\left|\rho_{j}-\lambda_{j}\right|$ in the classical limit. As an example consider the two-particle system where $\rho_{j}= \pm \rho$ and $\lambda_{j}= \pm \sqrt{E}$ are related by

$$
\sinh ^{2} \frac{\pi}{\hbar} \rho=\Delta(0) \sinh ^{2} \frac{\pi}{\hbar} \sqrt{E}
$$

Hence, for $\hbar \rightarrow 0$ we have $\rho=\sqrt{E}-\delta$ with $\delta \simeq 0.14$ for the classical ground-state energy $E=2$. However, this is the worst case. Both for larger energies in the two- particle system, and in systems with $N \gg 2$, the zeros of $\Delta$ are found to be very close to the $\lambda_{j}$. This shows that, despite the obvious similarity of the quantum approach to Kac and van Moerbeke's classical solution, the $\rho_{j}$ are not just the quantum analogs of the $\mu_{j}$. First of all there are, in general, $N$ different real solutions $\rho_{j}$ of $\Delta(\rho)=0$, while we had only $N-1$ classical variables $\mu_{j}$. Second, the values of the quantum variables $\rho_{j}$ are well outside the classical closed intervals to which the motion of $\mu_{j}$ is confined ( $\mu \equiv 0$ for $N=2, E=2$ ).

The proximity of the zeros $\rho_{j}$ and the poles $\lambda_{j}$ of $\Delta$ is very important in the practical use of Gutzwiller's scheme for the quantization of the Toda lattive, especially in the limit $\hbar \rightarrow 0$ where the rapid vanishing of $\Delta_{j}$ makes it very difficult to pinpoint zeros $\rho_{j}$ of $\Delta$. Luckily, the actual quantization conditions are expressed in terms of a phase function $\phi(\rho)$ evaluated at the $\rho_{j}$, and $\phi$ is slowly varying near each $\rho_{j}$.

The next step in this scheme, following Gutzwiller, is to calculate this phase factor $\phi(\rho)$ connecting the two bounded asymptotic expressions for the solution of Eq. (9):

$$
r_{j}(\kappa) \rightarrow \prod_{k}\left[\hbar^{ \pm i \kappa} \Gamma\left(1 \pm i\left(\kappa-\lambda_{k} / \hbar\right)\right)\right]^{-1}
$$

as $\kappa \rightarrow \rho / \hbar \mp i \infty$ ( $\Gamma$ is the usual $\Gamma$ function). This has to be done for each of the zeros $\rho_{j}$ of $\Delta$ giving $\phi_{j}=\phi\left(\rho_{j}\right)$ with

$$
\begin{aligned}
e^{2 i \phi(\rho)}=\prod_{k} & {\left[\hbar^{2 i \rho / \hbar} \frac{\Gamma\left(1+i\left(\rho-\lambda_{k}\right) / \hbar\right)}{\Gamma\left(1-i\left(\rho-\lambda_{k}\right) / \hbar\right)}\right] } \\
& \times \frac{r^{\prime \prime}(\rho)}{r^{\prime}(\rho)},
\end{aligned}
$$

where $r^{\prime}$ and $r^{\prime \prime}$ are semi-infinite-dimensional determinants given by

$$
r^{\prime}(\rho)=\left|\begin{array}{cccc}
1 & \frac{1}{D(\rho+i \hbar)} & & \\
\frac{1}{D(\rho+2 i \hbar)} & 1 & \frac{1}{D(\rho+2 i \hbar)} & \\
& \frac{1}{D(\rho+3 i \hbar)} & 1 & \frac{1}{D(\rho+3 i \hbar)}
\end{array}\right|
$$


and $r^{\prime \prime}(\rho)=\left[r^{\prime}(\rho)\right]^{*}$ for $\rho \in \mathbb{R}$. To our knowledge, there exists no analytic expression such as (13) for $r^{\prime}(\rho)$ but it is easily seen that $\phi(\rho)$ is well defined for arbitrary complex $\rho$ since the simple poles of $r^{\prime}$ at $\rho_{k, n}=\lambda_{k}-i n \hbar$, $n=1,2,3, \ldots$ cancel against the singularities of the $\Gamma$ functions at these points.

Numerical calculation of $\phi$ along the real axis reveals a very simple behavior (Fig. 1): The phase is a monotonically increasing function of $\rho$ which exhibits broad plateaus around $\rho=\lambda_{j}$ for sufficiently small $\hbar\left(\leq \frac{1}{10}\right.$, note that $\hbar$ is dimensionless in our units). The value of $\phi$ on these plateaus is a function of $\hbar$ and the conserved quantities (i.e., their eigenvalues) $\left\{\lambda_{j}\right\}$. Outside of the interval $\left[\lambda_{1}, \lambda_{N}\right]$ the semi-infinite determinants $r^{\prime}(\rho), r^{\prime \prime}(\rho)$ ap-
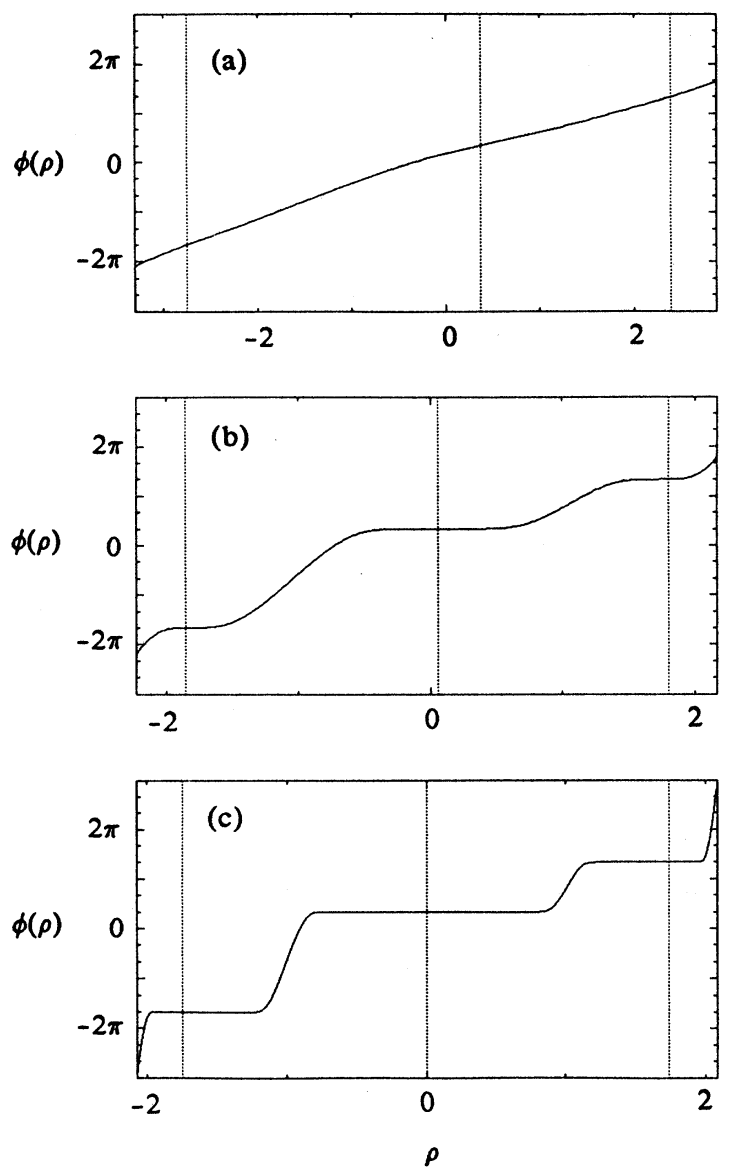

FIG. 1. Gutzwiller's phase function $\phi$ [Eq. (16)] vs $\rho$ in the first-excited state of the $N=3$ system [quantum numbers $\left.\left(n_{1} n_{2}\right)=(1,0)\right]$. (a) $\hbar=1: E=6.7114, A=2.4113$. (b) $\hbar=0.1$ : $E=3.348861, \quad A=0.179792$. (c) $\hbar=0.01$ : $E=3.034668$ 8, $A=0.0173872$. Dotted lines indicate the positions of the poles $\lambda_{j}$ of the Hill-type determinant $\Delta(12)$. The zeros $\rho_{j}$ of $\Delta-$ where the quantization condition (18) has to be fulfilled - are within the thickness of these lines (a); (b) and (c) the difference $\left|\rho_{j}-\lambda_{j}\right|$ is larger but well within the width of the plateaus of $\phi$. (Note the different scales on the $\rho$ axis.) proach unity giving $\phi \rightarrow \pm \infty$ determined by the phase of the $\Gamma$ functions in (16).

This simple functional dependence of $\phi$ on $\rho$ makes the quantization scheme numerically feasible: The existence of plateaus in $\phi$ makes the quantization condition insensitive to small variations of the quantum numbers $\rho_{j}$. Hence the precise determination of the zeros of $\Delta$ (which was not tractible numerically in the semiclassical regime as discussed above) is not necessary for the calculation of the $\phi_{j}$. The fact that $\phi(\rho)$ is approximately constant in a neighborhood of the pole $\lambda_{j}$ of $\Delta$ that includes the zero $\rho_{j}$ allows us to skip the first step of the quantization scheme and set $\phi_{j} \simeq \phi\left(\lambda_{j}\right)$ for values of $\hbar$ too small to determine $\rho_{j}$.

In the final step of Gutzwiller's quantization scheme for the periodic Toda chain the system parameters $\left\{\lambda_{j}\right\}$ have to be varied to fulfill certain conditions for the phases $\phi_{j}$.

By using the solution of the $(N-1)$-particle open chain with wave numbers corresponding to the zeros of the Hill determinant $\Delta$ as a basis for the eigenvalue problem of the $N$-particle closed one, the vanishing of the wave functions $\Psi\left(\left\{q_{j}\right\} ;\left\{\kappa_{j}\right\}\right)(8)$ is guaranteed whenever one of the $e^{q_{i+1}-q_{i}}(i=2, \ldots, N-1)$ becomes large. In addition, overall normalizability of the wave functions requires their boundedness for large $e^{q_{2}-q_{1}}$ or $e^{q_{1}-q_{N}}$. This can be obtained by suitable combination of the $\Psi$ 's with different assignments of the allowed $\kappa_{j}$ to the $q_{j}$. A tedious but straightforward calculation by Gutzwiller shows that a necessary condition for the cancellation of the exponentially divergent parts in the asymptotics of $\Psi$ is that the phase factors (16) are equal, i.e.,

$$
\phi_{1}=\phi_{2}=\cdots=\phi_{N}(\bmod \pi) \text {. }
$$

Since the $\rho_{j}$ are ordered $\rho_{1}<\rho_{2}<\cdots<\rho_{N}$, and $\phi(\rho)$ is monotonically increasing, this is equivalent to the condition $\phi_{j+1}-\phi_{j}=\pi\left(n_{j}+1\right)$ with non-negative integers $n_{j}$ (see Fig. 1).

Besides (18), there exists an identity which is fulfilled trivially for $N=2$ and $N=3$ and which was assumed to hold for $N>3$ by Gutzwiller:

$$
\sum_{j} \phi_{j}=0(\bmod \pi) \text {. }
$$

Together with Eq. (18) this restricts the allowed absolute values of $\phi_{j}$ to $(\pi v) / N$ with integer $v$ (with the same value of $v$ for all $j$ ).

We now turn to the problem of ground-state symmetry for an odd number of particles. In the quantization scheme above, one assumes implicitly that there are exactly $N$ real zeros of the Hill-type determinant $\Delta$ (12). However, as can be seen from the analytic expression (13) for $\Delta$, a necessary condition for this is that all the coefficients $K_{j}$ are nonzero.

A special situation arises in systems with an odd number of particles when one considers symmetric states, namely states with conserved quantities $(P=N \Lambda$ is the total momentum of the system):

$$
\lambda_{k}=2 \Lambda-\lambda_{N-k+1}, \quad k=1,2, \ldots, N .
$$


In this case $D(\rho)=-D(2 \Lambda-\rho)$, and it is straightforward to check that the singular terms in the expansion of $\Delta(\rho)$ for $\rho \simeq \lambda_{(N+1) / 2}=\Lambda$ cancel exactly. This leads to vanishing of the corresponding $K_{\Lambda}$ in the expression (13) for $\Delta$. Hence, there are only $N-1$ real values of $\rho$ fulfilling $\Delta(\rho)=0$. This observation was first noted by Gutzwiller for the $N=3$ system. $^{8} \mathrm{He}$ concluded that symmetric states do not exist in the three-particle periodic Toda lattice.

We want to reconsider this question of the existence of symmetric states in the odd- $N$ quantum periodic Toda chain since it is of some importance in understanding the whole quantization scheme. The naive guess for the ground state would be symmetric. If the quantization procedure rules out these states, then the ground state of the odd- $N$ quantum Toda chain must be degenerate, since a change from $\left\{\lambda_{j}\right\}$ to $\left\{-\lambda_{j}\right\}$ does not change the energy of the system. We argue here that this degeneracy does not occur - we believe the true ground state is symmetric.

There exist two limiting cases where the existence of symmetric states can be established.

(1) In the low-energy regime ( $E \simeq N$ for the $N$-particle system) the exponential interaction in the Toda Hamiltonian (1) can be expanded about the classical groundstate configuration $q_{n} \equiv$ const giving the $N$-particle harmonic chain to lowest order. In this limit the symmetric states become the eigenstates of the harmonic chain in which the modes with wave number $k$ and $2 \pi-k$ contain the same number of phonons. Since there is no obvious breaking of symmetry by inclusion of anharmonic terms this fact suggests that these states exist in the quantum Toda chain, too.

(2) In the semiclassical regime $\hbar \rightarrow 0$, and for large energies, the spectrum of the Toda chain can be obtained by Bohr-Sommerfeld quantization (see below). This approach gives a classification of states in terms of quantum numbers that are equivalent to the phonon numbers in the harmonic chain. Again, no indication for a quantum effect ruling out the existence of symmetric states can be found.

Finally, the Gutzwiller scheme as described above can be applied directly to the $N=3$ system. Our numerical results show that the phase function $\phi(\rho)$ has three plateaus for $\rho \simeq \lambda_{j}$ independent of the symmetry of the $\lambda_{j}$. To allow for symmetric states, the quantization condition (18) has to be used with $\phi_{N}=\phi\left(\rho_{N}\right)$, where

$$
\rho_{N}=\sum_{j=1}^{N} \lambda_{j}-\sum_{j=1}^{N-1} \rho_{j} \simeq \Lambda
$$

for states where $\Delta(\rho)=0$ has N-1 real solutions $\left\{\rho_{1}, \rho_{2}, \ldots, \rho_{N-1}\right\}$ only. [Note that this amounts to $(N-1) / 2$ independent quantization conditions only, since $\phi(\rho)=-\phi(2 \Lambda-\rho)(\bmod \pi)$ because of the symmetry of the $\lambda_{j}$. However, this is the number of independent parameters in the set $\left\{\lambda_{j}\right\}$ with (20).] Using (21), we find the Gutzwiller scheme gives complete agreement with the harmonic-chain results and the semiclassical results in the appropriate limits.

\section{BOHR-SOMMERFELD QUANTIZATION}

In the semiclassical regime $\hbar<<1$ and for high energies $E \gg E_{0}$ torus quantization is expected to give the spectrum of the Toda lattice. As has been discussed in Sec. II the Toda-Hamiltonian can be written, after separation of the center-of-mass motion, in terms of $N-1$ pairs of canonically conjugate variables $\left\{\mu_{m}, v_{m}\right\}$ where $\mu_{m}, v_{m}$ are related by Eq. (6). The periodic solutions of the dynamical equations for $\mu_{m}$ were restricted to motion in the $m$ th of the bounded intervals in which the characteristic polynomial $D$ of the Lax matrix [as defined by Eqs. (4)] fulfilled $D^{2}(\mu) \geq 4$. In terms of the original variables $\left\{p_{n}, q_{n}\right\}$, a small positive value of $D^{2}(\mu)-4$ at the $m$ th extremum of $D(\mu)$ versus $\mu$ can be understood as the excitation of a low-amplitude $m$ th harmonic wave. ${ }^{2}$ The relation (6) between $\nu_{m}$ and $\mu_{m}$ allows for the calculation of the action of these periodic orbits in $\mu-\nu$ space:

$$
\begin{aligned}
J_{m}=\oint v_{m} d \mu_{m} & =2 \int \operatorname{arccosh} \frac{|D(\mu)|}{2} d \mu \\
& =2\left|\int \frac{\mu D^{\prime}(\mu)}{\left[D^{2}(\mu)-4\right]^{1 / 2}} d \mu\right|,
\end{aligned}
$$

where the integration has to be performed over the $m$ th interval in which $|D(x)|>2$. Approximating $|D(\mu)|$ $\simeq D_{m}-B_{m}\left(\mu-\mu_{0}\right)^{2}$ in this region one obtains

$$
\begin{aligned}
& J_{m} \simeq 8\left(\frac{D_{m}+2}{B_{m}}\right)^{1 / 2}[K(k)-E(k)], \\
& k=\left(\frac{D_{m}-2}{D_{m}+2}\right)^{1 / 2},
\end{aligned}
$$

where $K$ and $E$ are the complete elliptic integrals of first and second kind, respectively. Semiclassical quantization in terms of these action variables is obtained by choosing values for the $\lambda_{j}$ such that

$$
J_{m}=2 \pi \hbar\left(n_{m}+\frac{1}{2}\right)
$$

with nonnegative integers $n_{m}$ being the quantum numbers of the system. Because of the identification of a periodic orbit in the pair of conjugate variables $\left(\mu_{m}, v_{m}\right)$ with the excitation of the $m$ th harmonic wave in the small-amplitude limit, these quantum numbers are the equivalent of phonon numbers in the $k=(2 \pi m) / N$ mode of the harmonic chain and may be interpreted as the numbers of anharmonic phonons (cnoidal waves in the classical limit) in the periodic Toda chain.

The neglect of higher terms in $D(x)$ for the derivation of Eq. (23) for $N>2$ limits its validity to systems near the (classical) ground state, namely

$$
\lambda_{j}=-2 \cos \pi \frac{2 j+1}{2 N} .
$$

For $N=2$, where no additional approximation is necessary to obtain (23) for the action $J_{m}$, numerical calculations show very good agreement between the spectrum obtained within this approach and the exact one obtained by using Gutzwiller's quantization scheme. The BohrSommerfeld quantization condition (24) can be solved or- 
TABLE I. Ground-state energy of the two-particle Toda lattice as obtained from the Gutzwiller scheme $\left(E_{0}\right)_{\text {exact }}$ and in Bohr-Sommerfeld quantization $\left(E_{0}\right)_{\text {BS }}$ for different values of $\hbar$.

\begin{tabular}{ccc}
\hline \hline$\hbar$ & $\left(E_{0}\right)_{\text {exact }}$ & $\left(E_{0}\right)_{\mathrm{BS}}$ \\
\hline 1.00 & 3.059173 & 3.030343 \\
0.50 & 2.515177 & 2.507695 \\
0.10 & 2.100621 & 2.100311 \\
0.05 & 2.050156 & 2.050078 \\
0.01 & 2.010006 & 2.010003 \\
\hline \hline
\end{tabular}

der by order in $\hbar$ giving for the ground-state energy

$$
E_{0} \simeq 2+\hbar+\frac{1}{32} \hbar^{2}+O\left(\hbar^{3}\right),
$$

where the first correction to the classical value of $E_{0}$ is the harmonic-chain result while the second one is due to anharmonic terms in the Toda potential. Comparison with the exact results obtained numerically within the Gutzwiller scheme shows that this is about half of the total anharmonic corrections to the ground-state energy (see Table I). For large energies the quantum fluctuations around the classical trajectories are known to be negligible. Hence Eq. (24) determines the asymptotic dependence of $E_{n}$ on the quantum number $n$ in the two-particle system:

$$
\sqrt{E_{n}-E_{0}} \ln \left(E_{n}-E_{0}\right) \propto n \quad(n>1) .
$$

As noted by Gutzwiller, ${ }^{8}$ the eigenenergies in the hyperbolic-cosine potential grow faster with $n$ than those of the harmonic oscillator $\left(E_{n} \propto n\right)$, but slower than those of a particle in a box $\left(\sqrt{E_{n}} \propto n\right)$.

\section{BETHE ANSATZ FOR THE TODA CHAIN}

As mentioned in the Introduction, the ground-state energy and the low-energy-excitation spectrum, ${ }^{5}$ as well as the free energy ${ }^{6}$ for the classical, infinite- $N$ Toda chain, can be obtained exactly by application of standard Bethe-ansatz analysis. This is quite surprising since, as discussed before, the exponential interaction in Eq. (1) is finite everywhere in the configuration space of the periodic chain. Furthermore, the analysis of Gaudin ${ }^{14}$ shows that, though the ladder operators for the algebraic Bethe ansatz can be constructed formally, they cannot be interpreted as creation or annihilation operators for physical excitations. Having verified the quantization conditions of Gutzwiller in various known limits, we now want to discuss their relation to the Bethe-ansatz approach.

The Bethe ansatz for the periodic Toda chain of length $L$ leads to the following set of nonlinear equations:

$$
k_{n} L=2 \pi I_{n}+\sum_{n \neq n^{\prime}} \delta\left(k_{n}-k_{n^{\prime}}\right)
$$

for $N$ wave numbers $k_{n}$ where the $I_{n}$ are a given set of distinct integers (for odd $N$ ) or half odd integers (for even $N)$. The appearance of $L$ is a peculiarity of the Betheansatz analysis. Equations (27) are obtained by imposing periodic boundary conditions on the wave function. This requires the introduction of absolute coordinates $q_{j} \rightarrow q_{j}+j(L / N)$ along the chain although, as should be clear from the above analysis, the Toda potential always leads to bound states - independent of the range of the $q_{j}$. However, the explicit $L$ dependence of the Betheansatz equations cancels against that introduced by the phase shift $\delta(k)$ when properly calculated from the asymptotic behavior of the wave function for a single particle in an $\exp (-x+L / N)$ potential: ${ }^{12}$

$$
\begin{aligned}
\delta(k) & =k\left(\frac{L}{N}-2 \ln \hbar\right)+i \ln \left(\frac{\Gamma(1+i k)}{\Gamma(1-i k)}\right) \\
& \equiv k \frac{L}{N}+\widetilde{\delta}(k) .
\end{aligned}
$$

With this expression Eqs. (27) reads

$$
0=2 \pi I_{n}+\frac{2 \pi v}{N}+\sum_{n \neq n^{\prime}} \widetilde{\delta}\left(k_{n}-k_{n^{\prime}}\right),
$$

where periodic boundary conditions have been used again to set $K=\sum_{n} k_{n}=2 \pi v / L$ with $v=0,1,2, \ldots, N-1$. In this formulation of the Bethe ansatz the independence of $L$ is guaranteed for the zero-total-momentum states.

There is an obvious formal similarity to the Gutzwiller quantization condition (18). Generalizing Eq. (16) to systems with nonzero total momentum $\Sigma_{n} \lambda_{n}$, and taking the logarithm, it can be written as

$$
0=2 \pi I_{n}+\frac{2 \pi v}{N}+\sum_{n^{\prime}} \widetilde{\delta}\left(\left(\rho_{n}-\lambda_{n^{\prime}}\right) / \hbar\right)+2 \phi_{s}\left(\rho_{n}\right),
$$

where $\phi_{s}$ is the phase of the semiinfinite determinant (17). The relative values of the integers $I_{n}$ can be given in terms of the anharmonic phonon numbers $n_{m}$ introduced in the preceding section by $I_{m+1}-I_{m}=n_{m}+1$.

Hence, neglecting $\phi_{s}$ and taking $\rho_{n}=\lambda_{n}$ the Gutzwiller phase condition is identical to the Bethe-ansatz equations with one difference: To obtain the term $2 \pi v / N$ in (29) we applied periodic boundary conditions on the Bethe-ansatz wave function to get $K=\sum_{n} k_{n}=2 \pi v / L$. In Eq. (30) the same term arises from condition (19), but is no longer related to the total momentum $\Sigma_{n} \lambda_{n}$. This means that the total density of states as given by the Bethe ansatz is too small by a factor of $N$ (Fig. 2).

Note that this does not affect the classical thermodynamics where the Bethe ansatz is known to give the exact result for the free energy - the factor $N$ in the density of states can be obtained by a renormalization of $\hbar \rightarrow \hbar / N$, becoming meaningless in the classical limit.

In the remainder of this section we want to discuss the approximations that have to be made to get the Betheansatz equations from Eq. (30). The replacement of $\rho_{n}$ by $\lambda_{n}$ (i.e., the identification of poles and zeros of the determinant $\Delta$ ) is justified in the semiclassical regime, as has been discussed above. The error introduced by the other assumption, namely that $\phi_{s}$ can be neglected, cannot be estimated because of the unknown analytic behavior of the semi-infinite determinants (17). For arbitrary $\rho$ this approximation is certainly not justified. Our numerical analysis shows that both $\phi_{s}$ and the phase generated by the sum involving the $\Gamma$ functions in $\widetilde{\delta}$ oscillate with amplitude roughly $\propto \hbar^{-1}$, and only their difference gives the 


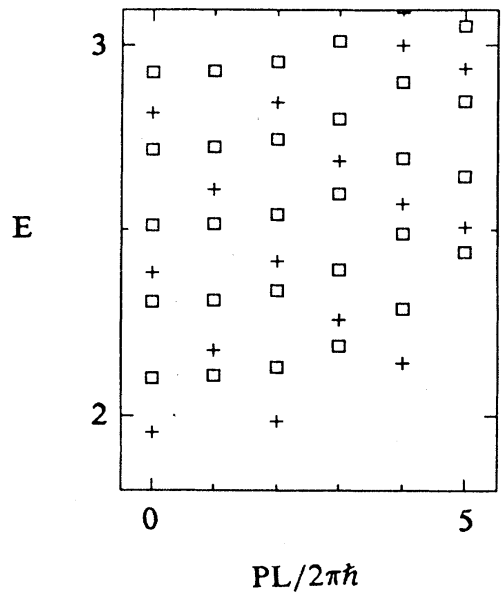

FIG. 2. Spectrum of the $N=2$ periodic Toda chain of length $L=4$ for $\hbar=0.1$ (energy $E$ vs total momentum $P$ in dimensionless units as defined in the text). Exact spectrum ( $\square)$ and Bethe-ansatz result $(+)$. The exact states with corresponding quantum numbers are the ones directly above the Bethe-ansatz states.

monotonically increasing function exhibiting plateaus near the poles of $\Delta$. On the other hand, we find that in systems with $N \gg 2 \phi_{s}$ vanishes at points very close to these poles.

This cancellation of corrections to the crude approximations on the Gutzwiller phase, which cannot be explained without a better understanding of the analytic properties of the phase $\phi_{s}$ [or of the semi-infinite determinants $r^{\prime}, r^{\prime \prime}$, Eq. (17)], suggests that the Bethe-ansatz equations (29) can indeed be used in the limit of large $N$ to calculate the spectrum of the zero-total-momentum states in the periodic quantum Toda lattice where the exact quantization conditions (18) are difficult to handle. The relation between the energy and the set of wave numbers $k_{n}$, however, is different from the one used in the standard Bethe ansatz:

$$
E=\frac{\hbar^{2}}{2}\left[\sum_{n}\left(k_{n}\right)^{2}-K^{2} / N\right],
$$

where $K=\sum_{n} k_{n}=2 \pi v / L$. States with nonzero total momentum $P=\hbar Q$ can be obtained by a uniform shift $\left\{k_{n}\right\} \rightarrow\left\{k_{n}+Q / N\right\}$. Periodic boundary conditions quantize $Q$ to values $2 \pi n / L$ with integer $n$ [being independent of the integer $v$ in (29)].

To get a quantitative estimate of the error made by solving the Bethe-ansatz equations instead of the exact Eqs. (18) we shall first consider the classical ground state. The classical ground-state energy of the $N$-particle system is $E_{N}=N$ in our units. Taking the classical limit $\hbar \rightarrow 0$ of the Bethe-ansatz equations (29) with $p_{n}=\hbar k_{n}$, one obtains to leading order in $\hbar$ by application of Stirling's formula for the $\Gamma$ functions

$$
0=\sum_{n \neq n^{\prime}}\left(p_{n}-p_{n^{\prime}}\right)\left(\ln \left|p_{n}-p_{n^{\prime}}\right|-1\right)
$$

These equations can be solved analytically for $N=2$

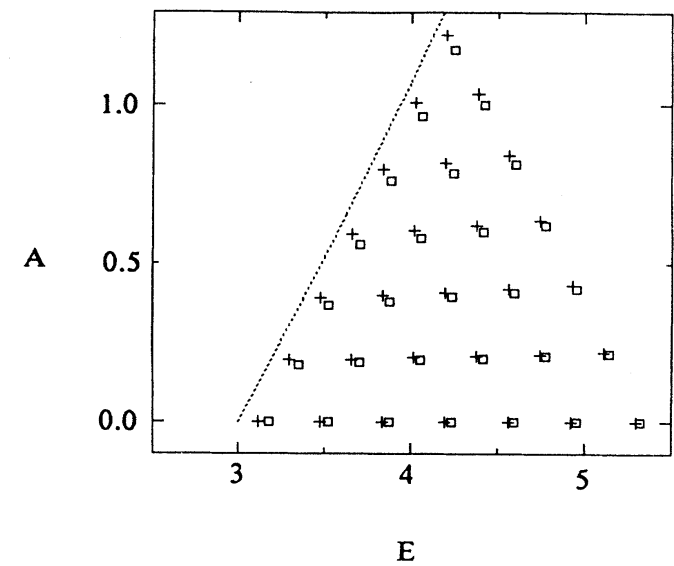

FIG. 3. Zero-momentum states of the $N=3$ periodic Toda chain for $\hbar=0.1$, constant of motion $A$ [see Eq. 4(b)] vs energy $E$ in dimensionless units as defined in text. Exact spectrum ( $\square$ ) and spectrum obtained by solution of the Bethe ansatz Eqs. (29) with $(31)(+)$. The dotted line indicates the border to the classical forbidden region. (Only states with $A \geq 0$ are shown, however, the spectrum is invariant under $A \leftrightarrow-A$.)

and $N=3$ by assuming $p_{n}= \pm p(0, \pm p)$ for the groundstate configuration. For $N=2$ one obtains $p=e / 2$ which gives an energy

$$
E_{2}=\frac{1}{2} \sum_{n} p_{n}^{2}=\frac{e^{2}}{4} \simeq 1.867 \text {. }
$$

For $N=3$ we find $p=e /\left(2^{2 / 3}\right)$ which gives $E_{3}=e^{2} /$ $\left(4^{2 / 3}\right) \simeq 2.932$ for the energy. Both results are significantly below the exact values for $E_{N}$. For $N>3$ (32) is a transcendental equation in the $p_{n}$ which can be solved numerically. Doing so for $N$ up to 100 we find that the Bethe ansatz gives

$$
\left(E_{N}\right)_{\mathrm{BA}}=N\left(1-\alpha_{N}\right) \text {, }
$$

where $\quad \alpha_{N} \simeq 0.601 / N^{3}+0.127 / N^{5}$ asymptotically for large $N$.

To check the accuracy of the energy of excited states as given by the Bethe ansatz against the exact results one has to consider finite $\hbar$. For $\hbar=\frac{1}{10}$ we have used both Gutzwiller's quantization scheme and the Bethe-ansatz equations with Eq. (31) to calculate the spectra for $N=2$ up to $E \simeq 125$ (corresponding to $n=300$ ) and for $N=3$ up to $E \simeq 5.3$ [corresponding to $\left(n_{1}, n_{2}\right)=(6,6)$, see Fig. 3]. The Bethe-ansatz results are getting closer to the exact ones as each of the quantum numbers $n_{j}$ gets large. Considering the energy $E$ only, we find that the difference between the exact and the Bethe-ansatz results decreases with increasing $E$. However, this decrease is very slow. As far as we can tell from our results, the asymptotic error of the Bethe ansatz is greater than $O(1 / E)$, probably containing a logarithmic $E$ dependence.

\section{ACKNOWLEDGMENTS}

H. F. gratefully acknowledges financial support by the Deutsche Forschungsgemeinschaft. 
${ }^{1}$ M. Toda, J. Phys. Soc. Jpn. 22, 431 (1967).

${ }^{2}$ M. Toda, Phys. Rep. 18, 1 (1975); Theory of Nonlinear Lattices (Springer-Verlag, Berlin, 1981).

${ }^{3}$ M. A. Olshanetsky and A. M. Perelomov, Phys. Rep. 94, 313 (1983).

${ }^{4}$ K. Sasaki, Phys. Rev. B 35, 3445 (1987).

${ }^{5}$ B. Sutherland, Rocky Mount. J. Math. 8, 413 (1978).

${ }^{6}$ M. Opper, Phys. Lett. 112A, 201 (1985).

${ }^{7}$ M. C. Gutzwiller, Ann. Phys. (N.Y.) 133, 304 (1981).

${ }^{8}$ M. C. Gutzwiller, Ann. Phys. (N.Y.) 124, 347 (1980).

${ }^{9}$ H. Flaschka, Phys. Rev. B 9, 1924 (1974).

${ }^{10}$ M. Kac and P. van Moerbeke, Proc. Nat. Acad. Sci. U.S.A. 72, 1627 (1975); 72, 2879 (1975).
${ }^{11}$ T. Shirafuji, Prog. Theor. Phys. Suppl. 59, 126 (1976).

${ }^{12}$ F. G. Mertens, Z. Phys. B 55, 353 (1984).

${ }^{13}$ B. Sutherland, J. Math. Phys. 12, 246 (1971); 12, 251 (1971); Phys. Rev. A 4, 2019 (1971); ibid. 5, 1372 (1971).

${ }^{14}$ M. Gaudin, La fonction d'onde de Bethe (Masson, Paris, 1983), Chap. 14.

${ }^{15}$ E. K. Sklyanin, in Nonlinear Equations in Classical and Quantum Field Theory, Vol. 226 of Lecture Notes in Physics, edited by N. Sanchez (Springer-Verlag, Berlin, 1985).

${ }^{16}$ N. W. McLachlan, Theory and Application of Mathieu Functions (Dover, New York, 1964).

${ }^{17} \mathrm{E}$. T. Whittaker and G. N. Watson, A Course of Modern Analysis (Cambridge University Press, Cambridge, 1962). 